\title{
Esophagus and Esophagogastric Junction Cancer pT1a TNM Finding v7
}

National Cancer Institute

\section{Source}

National Cancer Institute. Esophagus and Esophagogastric Junction Cancer pT 1a TNM

Finding v7. NCI Thesaurus. Code C89730.

Esophagus and esophagogastric junction cancer with tumor invading lamina propria or muscularis mucosae. (from AJCC 7th Ed.) 